TRANSACTIONS OF THE

AMERICAN MATHEMATICAL SOCIETY

Volume 353, Number 2, Pages 585-608

S 0002-9947(00)02727-6

Article electronically published on October 23, 2000

\title{
CENTRAL EXTENSIONS AND GENERALIZED PLUS-CONSTRUCTIONS
}

\author{
G. MISLIN AND G. PESCHKE
}

\begin{abstract}
We describe the effect of homological plus-constructions on the homotopy groups of Eilenberg-MacLane spaces in terms of universal central extensions.
\end{abstract}

\section{INTRODUCTION}

Higher algebraic $K$-theory was introduced by Quillen [27] by means of the plusconstruction (a precursor of which goes back to Varadarajan [32, p. 368]). When applied to a space $X$, it yields a map $X \longrightarrow X^{+}$which quotients out the maximal perfect subgroup of $\pi_{1} X$ without changing the homology of $X$. In the case where $X=B \mathrm{GL}(R)$ is the classifying space of the general linear group of a ring $R$, $K_{n}(R):=\pi_{n}\left(K_{0}(R) \times X^{+}\right)$.

While this construction is readily described, its homotopy theoretic properties, especially its effect on homotopy groups, remain largely mysterious. General results in this direction are due to Kervaire [23. He discovered the universal central extension $\pi_{2} K(G, 1)^{+} \longmapsto \widetilde{P G} \rightarrow P G$, where $P G$ is the maximal perfect subgroup of $G$, and $\widetilde{P G}$ is a perfect group satisfying $H_{2}(\widetilde{P G} ; \mathbb{Z})=0$. From this one easily deduces the natural isomorphisms $\pi_{2} K(G, 1)^{+} \cong H_{2}(P G ; \mathbb{Z})$ and $\pi_{3} K(G, 1)^{+} \cong H_{3}(\widetilde{P G} ; \mathbb{Z})$ which are special cases of our main theorem.

A homotopy theoretic environment which is suitable for the study of plusconstructions has been provided by the works of Bousfield [5, 6, 7, 8] and Dror Farjoun [14]. Given a homology theory $h$, there is a colocalizing functor $A_{h} X \longrightarrow X$ which extracts from $X$ a universal $h$-acyclic cover of its $h$-acyclic essence: $A_{h} X$ is $h$-acyclic and $\operatorname{map}_{*}\left(A, A_{h} X\right) \rightarrow \operatorname{map}_{*}(A, X)$ is a weak homotopy equivalence for every $h$-acyclic space $A$. Dually, there is a localizing functor $X \longrightarrow X^{+h}$ which strips $X$ of its $h$-acyclic essence: $\operatorname{map}_{*}\left(A, X^{+h}\right)$ is weakly equivalent to a point, for every $h$-acyclic space $A$.

The composite $A_{h} X \longrightarrow X \longrightarrow X^{+h}$ forms a homotopy fibration and, sometimes, also a homotopy cofibration- e.g. when $h=H(-; \mathbb{Z})$; see [20, 2.5]. Meier 25] first associated a plus-construction with a choice of an ordinary homology theory. This process was expanded in [11].

Received by the editors April 14, 1998.

1991 Mathematics Subject Classification. Primary 19D06, 55P60, 55Q15.

Key words and phrases. Plus-constructions, localization, colocalization, central extension. Research partially supported by NSERC of Canada. 
The map $X \rightarrow X^{+h}$ induces an isomorphism in $h$-homology, but is usually not a homotopy equivalence. Thus the homotopy groups of $X$ must change to accommodate the reduction of $X$ by its $h$-acyclic essence. We endeavor to understand this change of homotopy groups, and this paper is a first step. Our approach builds on the following two pillars.

- The universal properties of the fibration $A_{h} X \rightarrow X \rightarrow X^{+h}$ should be reflected in the form of universal properties of appropriate segments within the long exact sequence of homotopy groups of this fibration.

- The fibration $A_{h} X \rightarrow X \rightarrow X^{+h}$ yields the $\Pi$-central fibration $\Omega X^{+h} \stackrel{\partial}{\rightarrow}$ $A_{h} X \rightarrow X$; i.e. all Whitehead products of the form $[\partial \alpha, \beta]$ vanish, where $\alpha \in \pi_{p} \Omega X^{+h}$ and $\beta \in \pi_{q} A_{h} X, p, q \geq 1$; see (7.7).

Accordingly, appropriate segments within the long exact sequence of homotopy groups of the fibration $\Omega X^{+h} \stackrel{\partial}{\longrightarrow} A_{h} X \rightarrow X$ are central extensions of groups with a certain universal property. This statement takes its purest form in the case where $X=K(G, n)$ and $G$ is a group, abelian if $n \geq 2$, such that $\pi_{n} K(G, n) \longrightarrow$ $\pi_{n} K(G, n)^{+h}$ is the 0-map. For in this case, $\pi_{*} \Omega X^{+h} \longmapsto \pi_{*} A_{h} X \rightarrow \pi_{*} K(G, n)$ is an extension of $\Pi$-algebras, in the sense of Dwyer-Kan [19], which is central.

Here, we use this platform to gain insight into the effect of $+h$-localization on the homotopy groups of higher Eilenberg-MacLane spaces and on the second and third homotopy groups of $K(G, 1)$ 's. We summarize our main results in the following theorem.

Theorem. For $n \geq 1$ and a group $G$, abelian if $n \geq 2$, there is a unique maximal subgroup $P_{n}^{h} G$ of $G$ with the property that $\pi_{n} K\left(P_{n}^{h} \bar{G}, n\right) \rightarrow \pi_{n} K\left(P_{n}^{h} G, n\right)^{+h}$ is the 0-map. Moreover, the following hold.

(i) The $(n+1)$-st homotopy group of $K(G, n)^{+h}$ fits into the central extension

$$
\pi_{n+1} K(G, n)^{+h} \longleftrightarrow \widetilde{P_{n}^{h} G} \longrightarrow P_{n}^{h} G,
$$

which is universal in the sense explained in section 4. If $G$ is abelian, this identifies $\pi_{n+1} K(G, n)^{+h}$ as the representing object for the functor

$$
\operatorname{Ext}\left(P_{n}^{h} G,-\right) \cong H^{2}\left(P_{n}^{h} G ;-\right)
$$

on the category of all those groups $L$ for which $K(L, n+1)$ is $+h$-local.

(ii) If $n=1$, we have

$$
\begin{aligned}
\pi_{1} K(G, 1)^{+h} \cong G / P_{1}^{h} G \\
\pi_{2} K(G, 1)^{+h} \cong \pi_{2} K\left(P_{1}^{h} G, 1\right)^{+h} \cong H_{2}\left(K\left(P_{1}^{h} G, 1\right)^{+h} ; \mathbb{Z}\right), \\
\pi_{i} K(G, 1)^{+h} \cong \pi_{i} K\left(P_{1}^{h} G, 1\right)^{+h}, \quad i \geq 2 .
\end{aligned}
$$

Further, if $h$ is $\pi_{2}$-compatible (see (2.18)), then

$$
\pi_{3} K(G, 1)^{+h} \cong H_{3}\left(K\left(\widetilde{P_{1}^{h} G}, 1\right)^{+h} ; \mathbb{Z}\right) .
$$

For abelian $G$, our results overlap with [29] and [12]. However, their approaches are derived from a different viewpoint.

This paper is organized as follows. Section 0 contains background material on (co)localization. Section 1 establishes key results on maps whose homotopy fiber is $h$-acyclic. In section 2 , we introduce $h n$-perfect groups together with their duals, 
$h n$-acyclically reduced groups, and present their basic properties. Section 3 introduces, for $n \geq 1$, a localization functor of abelian groups from the effect of $h$-localization on abelian $K(G, n)$ 's. All of this is used in section 4 to formulate and prove our main results, stated above. Section 5 contains examples. Section 6 consists of an algebraic lemma. Section 7 presents prerequisites on $\Pi$-central fibrations which motivate our approach but are also of independent interest.

Acknowledgements. We thank Jérôme Scherer and the referee for many useful comments. The second author is grateful to the ETH (Zürich) for inviting him for a brief visit to facilitate completing this work.

\section{Preliminaries}

Here we gather some concepts and facts about homological localization which are relevant in what follows. Most of the key insights here are work of Bousfield. A good exposition can also be found in [29].

Homotopical localization with respect to a based map $f: U \rightarrow V$ of CW-spaces is a continuous homotopically idempotent functor $L_{f}$ on the category of compactly generated Hausdorff spaces; see [16, 14] and compare 6], [21. One may occasionally have need to resort to $\mathrm{CW}$-substitutes to avoid pathologies in mapping spaces, or to ensure the existence of covering maps. Alternatively, one can work simplicially. We assume that our spaces are based. Maps are based unless we specify otherwise.

The $L_{f}$-local objects $Z$, also called $f$-local objects, are characterized by the property that, between spaces of free maps, $f^{*}: \operatorname{map}(V, Z) \longrightarrow \operatorname{map}(U, Z)$ is a weak homotopy equivalence. A function $u: A \rightarrow B$ is an $L_{f}$-equivalence if $L_{f}(u)$ is a (weak) homotopy equivalence. If $c: W \rightarrow *$ is the map to a 1-point space, we write $X / / W$ for $L_{c} X$, and call it the $W$-reduction of $X$. Other notation in use includes $L_{W} X$ and $P_{W} X$. A $(W \rightarrow *)$-local space is also called $W$-reduced.

We consider only non-trivial homology theories $\left(\widetilde{h}_{*} \mathrm{~S}^{0} \neq 0\right)$, which can be described by a CW-spectrum. Such theories are additive. Therefore $h$-acyclic CWspaces $\left(h_{*}(X) \stackrel{\cong}{\rightrightarrows} h_{*}(\mathrm{pt})\right)$ are necessarily connected.

0.1. Proposition and Terminology. Given a homology theory h, there exists an $h$-equivalence $f$ between connected $C W$-spaces such that the following hold.

(i) $X \rightarrow L_{f} X=: X^{h}$ is Bousfield's homology localization with respect to $h$; see [5]. $X$ is called $h$-local if $X$ is $L_{f}$-local

(ii) If $A:=\operatorname{cofib}(f)$, then the $h$-localizing map $X \rightarrow X^{h}$ factors uniquely through the $h$-acyclic reduction $X^{+h}:=L_{A} X$, which we call the $+h$-construction of $X$; compare [8.4.4]. A space $Y$ is called $+h$-local or h-acyclically reduced, if $Y \simeq Y^{+h}$. A map $u$ is a + -equivalence if $L_{A}(u)$ is a (weak) homotopy equivalence. The $+h$-localization of a disconnected $C W$-space $X$ is given by the disjoint union of the $+h$-localizations of the connected components of $X$.

(iii) The homotopical colocalization of $X$ with respect to $A$ is the functor $u$ : $A_{h} X:=C W_{A} X \rightarrow X$ of Dror Farjoun [14]. It is characterized by the property that $A_{h} X$ is $h$-acyclic and that $u_{*}: \operatorname{map}_{*}\left(B, A_{h} X\right) \longrightarrow \operatorname{map}_{*}(B, X)$ is a weak homotopy equivalence for every $h$-acyclic space $B$.

(iv) For every connected space $X, A_{h} X \rightarrow X \rightarrow X^{+h}$ is a homotopy fibration.

Proof. Only (iv) remains to be shown. Let $F \rightarrow X \rightarrow X^{+h}$ be the homotopy fibration associated to $+h$-localization. By design, $\operatorname{map}_{*}\left(B, X^{+h}\right) \simeq *$ for every 
$h$-acyclic space $B$. Consequently, $\operatorname{map}_{*}(B, F) \longrightarrow \operatorname{map}_{*}(B, X)$ is a weak homotopy equivalence. To see that $F$ is $h$-acyclic we consider the map of fibrations below [18] p. 74]:

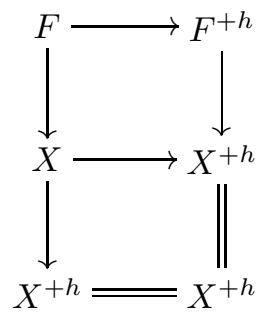

Visibly $F^{+h} \simeq *$, implying that $F$ is $h$-acyclic. From the universal property of $A_{h}$-colocalization we infer $F \simeq A_{h} X$.

The loop space operation relates $h$-localization and $+h$-localization as follows.

0.2. Lemma. If $X$ is + h-local and path connected, then $\Omega X$ is $h$-local.

Proof. Fiberwise $h$-localization gives the morphism of fibrations

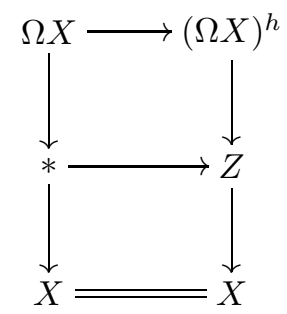

The $\operatorname{map} * \rightarrow Z$ is an $h$-equivalence. So $Z$ is $h$-acyclic. On the other hand, $Z$ is $+h$-local, being the total space of a fibration whose fiber and base are $+h$-local. Thus $Z \simeq *$, and the two fibrations are isomorphic.

For an arbitrary space $W$, the $W$-reduction map $X \rightarrow X / / W$ commutes with the covering space operation in the following precise sense.

0.3. Lemma. Given connected $C W$-complexes $W$ and $X$, let $K$ be the kernel of $\pi_{1} X \rightarrow \pi_{1}(X / / W)$. Then there is a natural homeomorphism

$$
\bar{X} / / W \longrightarrow \widehat{X / / W}
$$

where $\bar{X}$ is the covering space of $X$ corresponding to $K \triangleleft \pi_{1} X$ and $\widetilde{X / / W}$ is the universal cover of $X / / W$.

Proof sketch. Recall that $W$-reduction of a space $Y$ is constructed by repeating the pushout construction

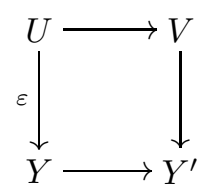


where the top arrow is

$$
\coprod_{k \geq 0}\left(\operatorname{map}\left(\mathrm{S}^{k} \rtimes W, Y\right) \times\left(\mathrm{S}^{k} \rtimes W\right)\right) \longrightarrow \coprod_{k \geq 0}\left(\operatorname{map}\left(\mathrm{S}^{k} \rtimes W, Y\right) \times C\left(\mathrm{~S}^{k} \rtimes W\right)\right),
$$

and $\varepsilon$ is evaluation. Thus $\pi_{1} Y \rightarrow \pi_{1} Y^{\prime}$ is onto. If $\kappa$ is a normal subgroup of $\pi_{1} Y$ containing $\operatorname{ker}\left(\pi_{1} Y \rightarrow \pi_{1} Y^{\prime}\right)$ let $\bar{Y}$ be the covering space corresponding to $\kappa$. Then

$$
\coprod_{k \geq 0} \operatorname{map}\left(\mathrm{S}^{k} \rtimes W, \bar{Y}\right) \longrightarrow \coprod_{k \geq 0} \operatorname{map}\left(\mathrm{S}^{k} \rtimes W, Y\right)
$$

is a covering map with $\pi_{1} Y / \kappa$ sheets. The morphism of pushout diagrams

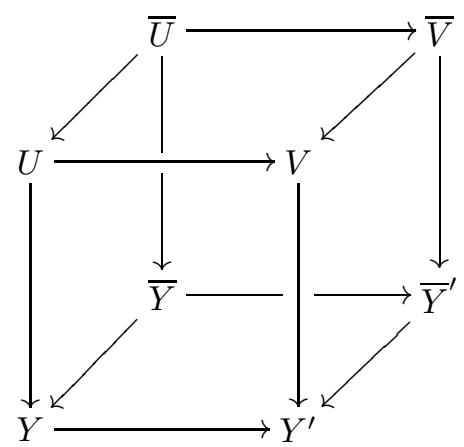

has $\bar{Y}^{\prime}$ connected, which covers $Y^{\prime}$ with $\pi_{1} Y / \kappa$ sheets. Moreover,

$$
\pi_{1} \bar{Y}^{\prime} \cong \kappa / \operatorname{ker}\left(\pi_{1} Y \rightarrow \pi_{1} Y^{\prime}\right) .
$$

Thus $\bar{Y}^{\prime} \rightarrow Y^{\prime}$ is the covering map of $Y^{\prime}$ corresponding to $\operatorname{im}\left(\kappa \rightarrow \pi_{1} Y^{\prime}\right)$. To infer the lemma, set $\kappa:=K$ and repeat this argument (transfinitely often if necessary).

0.4. Corollary. The higher homotopy groups of $K(G, 1) / / W$ depend only on the kernel of $\pi_{1} K(G, 1) \rightarrow \pi_{1} K(G, 1) / / W$.

0.5. Corollary. Given a connected $C W$-space $W$ and an arbitrary group $G$, then $K(G, 1)$ is $W$-reduced if and only if $\operatorname{Hom}\left(\pi_{1} W, G\right)=*$.

Proof. If $K(G, 1)$ is $W$-reduced, then $\operatorname{map}_{*}(W, K(G, 1))=*$ and so $\operatorname{Hom}\left(\pi_{1} W, G\right) \simeq$ *. Conversely, if $\operatorname{Hom}\left(\pi_{1} W, G\right)=*$, then $\pi_{1} K(G, 1) \longrightarrow \pi_{1} K(G, 1) / / W$ is an isomorphism. But then, with the notation of $(0.3), * \simeq \overline{K(G, 1)} \simeq \overline{K(G, 1)} / / W \simeq$ $K(\widetilde{G, 1)} / / W$. Thus $K(G, 1) \rightarrow K(G, 1) / / W$ is a homotopy equivalence.

We will need to recognize $h$-acyclic spaces. If $h$ is connective (i.e. $h_{n}(\mathrm{pt})=0$, for $n$ sufficiently small), we are aided by the following result of Bousfield [4].

0.6. Theorem. Given a connective homology theory h, let $P$ denote the set of primes $p$ for which $h_{*}(p t)$ is not uniquely p-divisible. Let

$$
R(h):= \begin{cases}\bigoplus_{p \in P} \mathbb{Z} / p & \text { if } h_{*}(p t) \text { is torsion, } \\ \mathbb{Z}_{P} & \text { if } h_{*}(p t) \text { is not torsion. }\end{cases}
$$

Then a space $X$ is h-acyclic if and only if $\widetilde{H}_{*}(X ; R(h))=0$.

More generally, we have 
0.7. Lemma. For a homology theory $h$, define $R(h)$ as in (0.6). Then every $H(-; R(h))$-acyclic space is also $h$-acyclic. In particular, $+h$-localization factors through $+H(-; R(h))$-localization.

Proof. If $Z$ is $H(-; R(h))$-acyclic, then the second page of the Atiyah-Hirzebruch spectral sequence for $h_{*} Z$ consists only of the 0 -th column. The claim follows.

A convenient tool for relating reduction and localizing functors of spaces is the following lemma of Zabrodsky; compare [31.

0.8. Lemma. Given a fibration $F \rightarrow E \stackrel{f}{\longrightarrow} B$, if a space $Y$ is $F$-reduced, then $Y$ is f-local.

0.9. Corollary. Suppose $\Omega$ is an h-acyclic loop space. Then $B \Omega$ is h-acyclic.

Proof. We have the fibration $\Omega \rightarrow * \stackrel{f}{\longrightarrow} B \Omega$. If $Y$ is arbitrary $+h$-local, then $Y$ is $\Omega$-reduced and, therefore, $f$-local; (0.8). Thus $f$ is a $+h$-equivalence, implying that $B \Omega$ is $h$-acyclic.

\section{1. $h$-ACYCLIC MAPS}

1.1. Definition. A map of path connected spaces $f: X \rightarrow Y$ is $h$-acyclic if fib $(f)$ is $h$-acyclic.

Our definition of " $h$-acyclic map" extends Quillen's in [28]. We suggest thinking of an $h$-acyclic map as being $h$-acyclicity reducing, because the target of an $h$ acyclic map appears in a universal way between $X$ and the completely $h$-acyclicity reduced space $X^{+h}$. This is one possible interpretation of the lemma below.

1.2. Lemma. Associated with an h-acyclic map $f: X \rightarrow Y$ there is the natural commutative diagram below whose rows and columns are fibrations:

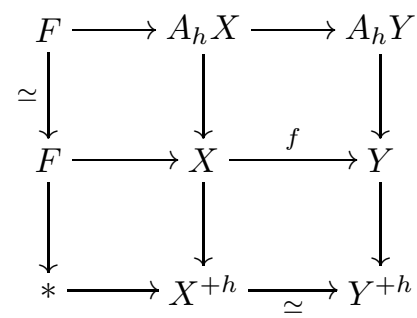

Proof. Universality of the operations $A_{h}$ and ${ }^{+h}$ yields the morphism of the two vertical fibrations on the right. From Zabrodsky's lemma (0.8) or fiberwise localization [14, 1.H.1], we see that $f$ is a $+h$-equivalence. Therefore $X^{+h} \rightarrow Y^{+h}$ is a homotopy equivalence, and, hence, the homotopy fibers of the horizontal maps are as indicated.

1.3. Corollary. An $h$-acyclic map is a + -equivalence and, hence, an $h$-equivalence.

Based on this information we expect

1.4. Corollary. If $X$ is path connected, then the universal map $X^{+h} \rightarrow X^{h}$ is a homotopy equivalence if and only if $\mathrm{fib}\left(X \rightarrow X^{h}\right)$ is h-acyclic, which is the case if and only if $\mathrm{fib}\left(X^{+h} \rightarrow X^{h}\right)$ is $h$-acyclic. 
Proof. We verify one implication: Suppose $F:=\operatorname{fib}\left(X \rightarrow X^{h}\right)$ is $h$-acyclic. By (1.2), $X^{+h} \rightarrow\left(X^{h}\right)^{+h}=X^{h}$ is a homotopy equivalence. The rest of the argument is similar.

Thus an $h$-acyclic map is an $h$-equivalence with some additional property. We make this statement precise in the case where $h$ is connective.

1.5. Proposition. Let $h$ be a connective homology theory. Then a map $f: X \rightarrow Y$ of path connected spaces is $h$-acyclic if and only if $f$ induces an isomorphism in homology with twisted coefficients

- $f_{*}: \bigoplus_{P} \mathcal{H}_{*}\left(X ; f^{*} \mathbb{Z} / p\left[\pi_{1} Y\right]\right) \longrightarrow \bigoplus_{P} \mathcal{H}_{*}\left(Y ; \mathbb{Z} / p\left[\pi_{1} Y\right]\right)$, if $h_{*}(p t)$ is torsion,

- $f_{*}: \mathcal{H}_{*}\left(X ; f^{*} \mathbb{Z}_{P}\left[\pi_{1} Y\right]\right) \longrightarrow \mathcal{H}_{*}\left(Y ; \mathbb{Z}_{P}\left[\pi_{1} Y\right]\right)$, if $h_{*}(p t)$ is not torsion.

In either case, $P$ is the set of primes $p$ for which $h_{*}(p t)$ is not uniquely $p$-divisible.

Proof. This follows by combining Bousfield's result (0.6) with [20] or [25, 1.1].

1.6. Proposition. If $h$ is connective and $X^{h}$ is simply connected, then the natural map $u: X^{+h} \rightarrow X^{h}$ is a weak homotopy equivalence.

Proof. $u$ is an $h$-equivalence and, hence, an $H(-; R)$-equivalence; see (0.6). Further, $u$ is $h$-acyclic, by using (1.5) and the fact that $X^{h}$ is simply connected. Thus fib $(u)$ is $+h$-local and $h$-acyclic, hence is a point, which implies the claim.

1.7. Example. If $h$ is connective, then $+h$-localization and $h$-localization agree on simply connected spaces. For, in this case, $h$-localization does not reduce connectivity; see [7, 7.3].

The following example illustrates that $+h$-localization and $h$-localization can agree on Eilenberg-MacLane spaces, even if $h$ is not connective.

1.8. Example. If $h=K$ is real or complex $K$-theory, and $G$ is an abelian group, then

(i) $K(G, 1)^{+h}=K(G, 1)^{h}=K(G, 1)$,

(ii) $K(G, 2)^{+h}=K(G, 2)^{h}=K(G /$ torsion, 2),

(iii) $K(G, i)^{+h}=K(G, i)^{h}=K(G \otimes \mathbb{Q}, i)$, for $i \geq 3$.

Proof. $K(G, n)^{h}$ has been computed by Mislin; see [26]. Thus the claims will follow once we have shown that, in each case, $\operatorname{fib}\left(K(G, n)^{+h} \rightarrow K(G, n)^{h}\right)$ is $h$-acyclic. Indeed, $K(G, 1)^{h}=K(G, 1)$, implying (i). Moreover, $K(G, 2)^{h}=K(G /$ torsion, 2$)$ and, therefore, one has a fibration $K(\operatorname{torsion}(G), 2) \rightarrow K(G, 2) \longrightarrow K(G, 2)^{h}=$ $K\left(G /\right.$ torsion, 2) with an $h$-acyclic fiber. Thus $K(G, 2)^{h}=K(G, 2)^{+h}$. If $i \geq 3$, then

$$
F:=\operatorname{fib}\left(K(G, i) \longrightarrow K(G \otimes \mathbb{Q}, i)=K(G, i)^{h}\right)=K(B, i) \times K(C, i-1)
$$

with $B$ and $C$ torsion groups; see 7.2 Thus $K(B, i)$ and $K(C, i-1)$ are $h$-acyclic. Localization commutes with finite products; [18, p. 5]. Thus $F$ is $h$-acyclic.

1.9. Remark. If $h=H(-; \mathbb{Z})$, then the fibration $A_{h} X \rightarrow X \rightarrow X^{+h}$ is also a cofibration; [20, 2.5]. This is not so in general, as the following example illustrates.

Take $h:=H(-; \mathbb{Z} / p)$ and $X=K\left(\mathbb{Z}_{p^{\infty}}, 1\right)$. Then $X^{h}=K\left(\mathbb{Z}_{p}^{\wedge}, 2\right)$, because of the short exact sequence $\mathbb{Z}_{p}^{\wedge} \longmapsto \mathbb{Q}_{p}^{\wedge} \rightarrow \mathbb{Z}_{p^{\infty}}$, where $\mathbb{Z}_{p}^{\wedge}$ denotes the $p$-adic integers. As $X^{h}$ is 1-connected, $X^{h}=X^{+h}$ by (1.6). The fiber of $K\left(\mathbb{Z}_{p^{\infty}}, 1\right) \rightarrow K\left(\mathbb{Z}_{p}^{\wedge}, 2\right)$ is $K\left(\mathbb{Q}_{p}^{\wedge}, 1\right)$, where $\mathbb{Q}_{p}^{\wedge}$ denotes the $p$-adic numbers. But the cofiber $C$ of $K\left(\mathbb{Q}_{p}^{\wedge}, 1\right) \rightarrow$ 
$K\left(\mathbb{Z}_{p^{\infty}}, 1\right)$ is not $K\left(\mathbb{Z}_{p}^{\wedge}, 2\right)$, as one sees by comparing rationalizations: $K\left(\mathbb{Z}_{p}^{\wedge}, 2\right)_{\mathbb{Q}}=$ $K\left(\mathbb{Q}_{p}^{\wedge}, 2\right)$, which is different from $\Sigma K\left(\mathbb{Q}_{p}^{\wedge}, 1\right)=C_{\mathbb{Q}}$, because we have the cofiber sequence

$$
* \simeq K\left(\mathbb{Z}_{p^{\infty}}, 1\right)_{\mathbb{Q}} \rightarrow C_{\mathbb{Q}} \rightarrow \Sigma K\left(\mathbb{Q}_{p}^{\wedge}, 1\right) \rightarrow \Sigma K\left(\mathbb{Z}_{p^{\infty}}, 1\right)_{\mathbb{Q}} \simeq * .
$$

Thus, in general, $X \rightarrow X^{+h}$ factors through $\operatorname{cofib}\left(A_{h} X \rightarrow X\right)=: C$. But $C$ need not be $h$-acyclically reduced.

\section{2. $h n$-PERFECT AND $h n$-ACYCLICALLY REDUCED GROUPS}

Fix a homology theory $h$. Given an integer $n$ and a group $G$, abelian if $n \geq 2$, we have the fibration

$$
A_{h} K(G, n) \rightarrow K(G, n) \longrightarrow K(G, n)^{+h} .
$$

In order to explain the influence of the universal properties of these maps on the homotopy groups in the long exact sequence of this fibration we introduce the groups

$$
P_{n}^{h} G:=\operatorname{ker}\left(\pi_{n} K(G, n) \rightarrow \pi_{n} K(G, n)^{+h}\right)
$$

and

$$
Q_{n}^{h} G:=\text { (maximal quotient } Q \text { of } G \text { with } P_{n}^{h} Q \text { trivial). }
$$

As we shall see, the higher homotopy groups of $K(G, n)^{+h}$ depend only on $P_{n}^{h} G$; see (3.7).

2.1. Definition. Let $n \geq 1$. A group $G$, abelian if $n \geq 2$, is called $h n$-perfect if $P_{n}^{h} G=G$. Dually, $G$ is $h n$-acyclically reduced if $G=Q_{n}^{h} G$.

2.2. Example. Let us consider $h=H(-; \mathbb{Z})$. Then $P_{1}^{h} G$ is just the unique maximal perfect subgroup of $G$. Moreover, $G$ is $h 1$-perfect if and only if $G$ is perfect. After all, $G$ is perfect if and only if $K(G, 1)^{+}$is simply connected. Dually, $G$ is $h 1$ acyclically reduced exactly when its maximal perfect subgroup is trivial. Finally, every abelian group is $h n$-acyclically reduced for all $n \geq 1$.

In general, $G$ is $h n$-acyclically reduced if and only if $P_{n}^{h} G$ is trivial. We will discuss the case of $h=K$ (real or complex $K$-theory) below. We will show how to characterize $L:=\pi_{n+1} K(G, n)^{+h}$ via a certain universal central extension

$$
L \longmapsto A \rightarrow P_{n}^{h} G .
$$

Dually, we show how to characterize $A^{\prime}:=\pi_{n-1} A_{h} K(G, n)$ via a certain short exact sequence

$$
Q_{n}^{h} G \longmapsto L^{\prime} \rightarrow A^{\prime}
$$

whose universal properties are dual to those of the $L$-determining extension. Hence we speak of a universal coextension here.

Here are some basic properties of $h n$-perfect and $h n$-acyclically reduced groups, followed by some lemmas needed to verify these properties.

2.3. Proposition (Basic properties of $h n$-perfect groups). The following hold.

(i) The class of hn-perfect groups is closed under quotients, arbitrary colimits and weak products.

(ii) If $A$ is an $(n-1)$-connected $h$-acyclic space, then $\pi_{n} A$ is hn-perfect.

(iii) For every space $X, \operatorname{ker}\left(\pi_{1} X \rightarrow \pi_{1} X^{+h}\right)$ is h1-perfect. 
(iv) Every group $G$ has a unique maximal hn-perfect subgroup $P_{n}^{h} G$.

(v) If $G$ is abelian and $n \geq 1$, then $P_{n}^{h}\left(G / P_{n}^{h} G\right)=0$.

(vi) Let $h$ and $k$ be homology theories such that every $h$-equivalence is also a $k$ equivalence. Then, for each $n \geq 1$, hn-perfect groups are also kn-perfect.

2.4. Proposition. (Basic properties of $h n$-acyclically reduced groups) The following hold.

(i) The class of hn-acyclically reduced groups is closed under subgroups and arbitrary inverse limits.

(ii) Every group $G$ has a unique maximal hn-acyclically reduced quotient $Q_{n}^{h} G$. If $G$ is abelian then $Q_{n}^{h} G=\operatorname{coker}\left(\pi_{n} A_{h} K(G, n) \longrightarrow \pi_{n} K(G, n)\right)$. If $n=1$, then there is the possibly transfinite sequence of epimorphisms with

$$
Q_{1}^{h} G=\underline{\lim _{\longrightarrow}}\left\{G \rightarrow G / P_{1}^{h} G \rightarrow\left(G / P_{1}^{h} G\right) / P_{1}^{h}\left(G / P_{1}^{h} G\right) \rightarrow \cdots\right\} .
$$

(iii) Let $h$ and $k$ be homology theories such that every $h$-equivalence is also a $k$-equivalence. Then kn-acyclically reduced groups are also hn-acyclically reduced.

Here is the key lemma which is needed to understand the effect of $+h$-localization on $K(G, n)$ 's with $G$ abelian.

2.5. Lemma. For $n \geq 1$ and an abelian group $G$, there is the natural fiber sequence

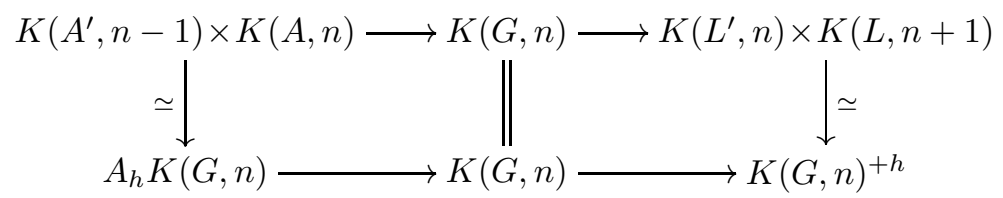

whose long exact sequence of homotopy groups consists of

$$
0 \rightarrow L \longmapsto A \rightarrow G \rightarrow L^{\prime} \rightarrow A^{\prime} \rightarrow 0 .
$$

Moreover, if $n=1$, then $A^{\prime}=0$.

Proof. According to [14, 4.12], $A_{h} K(G, n)$ is a GEM whose homotopy groups above dimension $n$ vanish. Further, $K(G, n)^{+h}$ is an $(n-1)$-connected GEM; see [15, 1.11]. The claim follows.

From (2.5) we deduce the following.

2.6. Corollary. For an arbitrary group $G$, abelian if $n \geq 2$, the canonical map $K(G, n) \rightarrow K(G, n)^{+h}$ is a homotopy equivalence if the induced map $\pi_{n} K(G, n) \rightarrow$ $\pi_{n} K(G, n)^{+h}$ is an isomorphism.

Proof. Suppose $\pi_{n} K(G, n) \rightarrow \pi_{n} K(G, n)^{+h}$ is an isomorphism. If $n \geq 2$ then, in the notation of (2.5), $A^{\prime}=0$ and $L \cong A$. Thus the $h$-acyclic space $K(A, n)$ is homotopy equivalent to the $h$-local space $\Omega K(L, n+1)$; see 0.2$)$. Thus $A=L=0$, which implies the claim.

If $n=1$, we have the equivalence

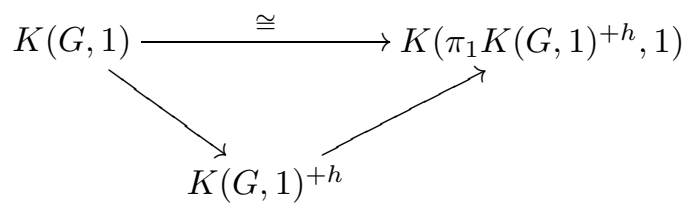


which exposes $K(G, 1)$ as a retract of the $+h$-local space $K(G, 1)^{+h}$. Thus $K(G, 1)$ is already $+h$-local, which implies the claim.

2.7. Corollary. A group $G$ is h1-acyclically reduced iff $K(G, 1)$ is + h-local.

2.8. Lemma. For an abelian group $G$ and $n \geq 1$ the following are equivalent.

(i) $G$ is hn-perfect.

(ii) $+h$-localization yields a fibration of the form:

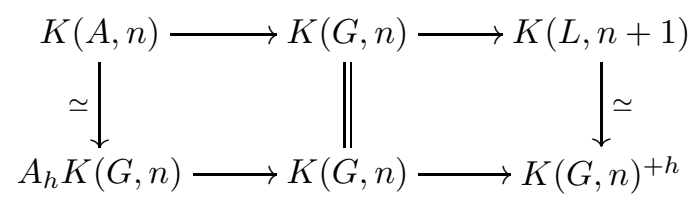

Proof. (i) $\Longrightarrow$ (ii) Since $G$ is $h n$-perfect, the exact sequence of homotopy groups from 2.5 takes the form $L \longmapsto A \rightarrow G \stackrel{0}{\longrightarrow} L^{\prime} \cong A^{\prime}$. Thus $L^{\prime} \cong A^{\prime}$, implying that the $h$-local space $K\left(L^{\prime}, n-1\right)$, see $(0.2)$, is a retract of the $h$-acyclic space $K\left(A^{\prime}, n-1\right) \times K(A, n)$. Therefore $K\left(L^{\prime}, n-1\right)$ is $h$-acyclic as well. But then $K\left(L^{\prime}, n-1\right)$ is a point, implying that $L^{\prime}=0$, as claimed.

(ii) $\Longrightarrow\left(\right.$ i) $\quad G$ is $h n$-perfect, since $\pi_{n} K(G, n)^{+h}=0$.

2.9. Corollary. If $G$ is hn-perfect, then $H_{1}(G ; \mathbb{Z})$ is $h(n+1)$-perfect.

Proof. We have the fibration $A_{h} K(G, n) \rightarrow K(G, n) \rightarrow K(G, n)^{+h}$, in which $A_{h} K(G, n)$ is $(n-1)$-connected and $K(G, n)^{+h}$ is $n$-connected. Suspending the first map yields the diagram $\Sigma A_{h} K(G, n) \rightarrow \Sigma K(G, n) \rightarrow K\left(H_{1}(G ; \mathbb{Z}), n+1\right)$ in which all spaces are $n$-connected. $\Sigma A_{h} K(G, n)^{+h}$ is $h$-acyclic and $\pi_{n+1} \Sigma A_{h} K(G, n)$ maps onto $\pi_{n+1} K\left(H_{1}(G ; \mathbb{Z}), n+1\right)$. Thus $H_{1}(G ; \mathbb{Z})$ is $h(n+1)$-perfect.

2.10. Lemma. Let $n \geq 1$ and $G$ a group, abelian if $n \geq 2$. Then the following are equivalent.

(i) $G$ is hn-acyclically reduced.

(ii) $+h$-localization yields a fibration of the form:

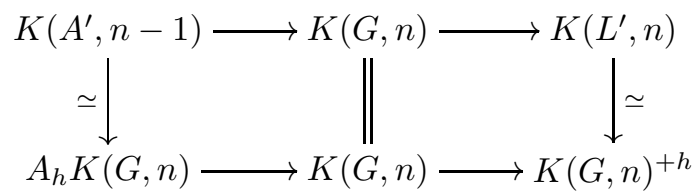

Proof. If $n=1$, the claim follows from (2.7). Thus suppose $n \geq 2$.

(i) $\Longrightarrow$ (ii) According to (2.5) the $+h$-localization fibration of an abelian group $G$ yields the exact sequence of groups $L \stackrel{\cong}{\longrightarrow} \stackrel{0}{\longrightarrow} \longrightarrow L^{\prime} \rightarrow A^{\prime}$. Considering that $P_{n}^{h} G=0$ we find that the arrows in this sequence behave as indicated. Thus the $h$-local space $K(L, n)$ is a retract of the $h$-acyclic space $K(A, n) \times K\left(A^{\prime}, n-1\right)$, implying that $L=0=A$.

(ii) $\Longrightarrow$ (i) In this situation, $\pi_{n} K(G, n) \rightarrow \pi_{n} K(G, n)^{+h}$ is a monomorphism, implying that $G$ is $h n$-acyclically reduced.

2.11. Corollary. A group $G$ is hn-acyclically reduced if and only if $[Y, K(G, n)]=$ 0 , for every $(n-1)$-connected $h$-acyclic space $Y$. 
Proof. The case $n=1$ follows from (0.5). If $n>1$, we have associated with every $h$-acyclic space $Y$ the isomorphism $\left[Y, A_{h} K(G, n)\right] \stackrel{\cong}{\longrightarrow}[Y, K(G, n)]$. If $G$ is $h n$ acyclically reduced, then $A_{h} K(G, n)$ is of the form $K\left(A^{\prime}, n-1\right)$ (2.10), implying that $\left[Y, A_{h} K(G, n)\right]=0$ for $(n-1)$-connected $Y$. Conversely, if $[Y, K(G, n)]=0$ for $(n-1)$-connected $h$-acyclic $Y$, then, in the notation of (2.5), $K(A, n) \rightarrow K(G, n)$ is null, implying that $A=L=0$. The claim follows from (2.10).

2.12. Lemma. For $n \geq 2$, if an abelian group $G$ is hn-acyclically reduced, then $G$ is $h(n-1)$-acyclically reduced.

Proof. The fibration $A_{h} K(G, n) \rightarrow K(G, n) \rightarrow K(G, n)^{+h}$ has the form

$$
K\left(A^{\prime}, n-1\right) \rightarrow K(G, n) \rightarrow K\left(L^{\prime}, n\right) ;
$$

see (2.10). Looping it gives us a monomorphism

$$
\pi_{n-1} K(G, n-1) \longmapsto \pi_{n-1} K\left(L^{\prime}, n-1\right) .
$$

But $K\left(L^{\prime}, n-1\right)$ is $h$-local by $(0.2)$. So $L^{\prime}$ is $h(n-1)$-acyclically reduced. $G$ is a subgroup of $L^{\prime}$, and the argument is complete by (2.4) $\mathrm{i}$ ).

Proof of proposition (2.3). (i) Closure under quotients. If $n=1$, we recall that the $+h$-construction is a coning construction, hence induces an epimorphism of fundamental groups, hence preserves epimorphisms of fundamental groups. Therefore the class of $h 1$-perfect groups is closed under quotients.

If $n \geq 2$, consider the $+h$-localization fibration of $K(G, n)$, namely

$$
K(A, n) \hookrightarrow K(G, n) \longrightarrow K(L, n+1) .
$$

Here we used the fact that $G$ is $h n$-perfect and (2.8),(i) $\Longrightarrow$ (ii). If $G / H$ is a quotient of $G$, we find a map from the $h$-acyclic space $K(A, n)$ to $K(G / H, n)$ inducing an epimorphism on $\pi_{n}$. The $+h$-localization of $K(G / H, n)$ factors through the cofiber of this map, implying that $G / H=P_{n}^{h}(G / H)$.

Closure under colimits. Every colimit of groups is a quotient of the coproduct of those groups which occur in the colimit diagram. (If $n=1$, "coproduct" means "free product". If $n>1$, "coproduct" means "direct sum".) Thus it suffices to show that $\mathcal{C}_{n}$ is closed under coproducts. A coproduct $\bigsqcup G_{\lambda}$ of $h n$-perfect groups arises as $\pi_{n}$ of the wedge $\bigvee_{\lambda} K\left(G_{\lambda}, n\right)$. Now $\bigvee_{\lambda} A_{\lambda}$ is $h$-acyclic and $\pi_{n}\left(\bigvee A_{\lambda} \rightarrow \bigvee K\left(G_{\lambda}, n\right)\right)$ is onto. Thus $\pi_{n} K\left(\bigsqcup G_{\lambda}, n\right)^{+h}=0$, implying that $\bigsqcup G_{\lambda}$ is $h n$-perfect.

Closure under weak products. The $+h$-construction commutes with finite products [17, Theorem 4], and a finite product of $h$-acyclic spaces is again $h$-acyclic. Therefore the class $\mathcal{C}_{n}$ of $h n$-perfect groups is closed under finite products. The weak product is a directed colimit of finite products. Now use the fact that $h n$ perfect groups are closed under colimits.

(ii) By hypothesis, $A^{+h} \simeq *$. Thus $P_{n}^{h} \pi_{n} A=\pi_{n} A$.

If $n>1$, the technique used in (i) shows that $K\left(\pi_{n} A, n\right)^{+h} \simeq K(L, n+1)$. Thus $\pi_{n} A$ is $h n$-perfect.

(iii) $\operatorname{ker}\left(\pi_{1} X \rightarrow \pi_{1} X^{+h}\right)$ is a quotient of the $h 1$-perfect group $\pi_{1} A_{h} X$. The claim follows from (i).

(iv) $\operatorname{ker}\left(\pi_{n} K(G, n) \rightarrow \pi_{n} K(G, n)^{+h}\right)$ is a quotient of $\pi_{n}$ of an $(n-1)$-connected $h$-acyclic space. By (ii) this kernel is $h n$-perfect. On the other hand, every $h n$ perfect subgroup $H$ of $G$ is contained in $P_{n}^{h} G$. This can be read off the morphism 
of fibrations below:

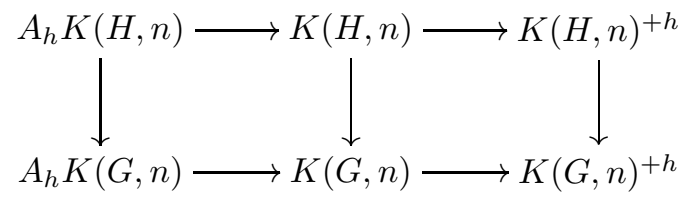

(v) Let $K(G, n) \rightarrow K\left(L^{\prime}, n\right) \times K(L, n+1)$ be the $+h$-localizing map; see (2.5). In any case $B:=P_{n}^{h}\left(G / P_{n}^{h} G\right)<L^{\prime}$. If $B \neq 0$, there exists an essential map from an $h$-acyclic space into $K(G, n)^{+h}$ - a contradiction.

(vi) holds because $+k$-localization factors through $+h$-localization and does not decrease connectivity of Eilenberg-MacLane spaces.

The proof of (2.3) is complete.

Proof of proposition (2.4). (i) If $G$ is $h n$-acyclically reduced, let $G^{\prime}<G$ be a subgroup. Then we have a short exact sequence $\pi_{n} A_{h} K\left(G^{\prime}, n\right) \rightarrow G^{\prime} \longmapsto \pi_{n} K\left(G^{\prime}, n\right)^{+h}$, where the arrow on the right is a monomorphism because $\operatorname{im}\left(\pi_{n} A_{h} K\left(G^{\prime}, n\right) \rightarrow\right.$ $\left.G^{\prime}\right)<\operatorname{ker}\left(G \rightarrow Q_{n}^{h} G\right)=0$. Thus $G^{\prime}$ is $h n$-acyclically reduced.

Let $G:=\lim \left\{G_{\lambda}\right\}$ be the inverse limit of a system of $h n$-acyclically reduced groups. If $Y \overleftarrow{\text { is }}$ an $h$-acyclic space, then $\left[Y, K\left(G_{\lambda}, n\right)\right]=*$ for each $\lambda$; see (2.11). Thus $\prod G_{\lambda}$ is $h n$-acyclically reduced. $G$ is a subgroup of $\prod G_{\lambda}$. The claim follows.

(ii) Let $Q:=\operatorname{im}\left(\pi_{n} K(G, n) \rightarrow \pi_{n} K(G, n)^{+h}\right)$. For an arbitrary $h n$-acyclically reduced quotient $G^{\prime}$ of $G$, we have the commutative diagram below; see (2.10):

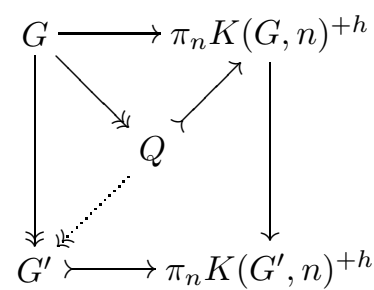

Thus $G^{\prime}$ is a quotient of $Q$. If $n \geq 2, Q$ is $h n$-acyclically reduced by (2.10), in which case the claim follows. If $n=1$, we have the tower of $h_{1}$-acyclic reductions

$$
G \rightarrow G / P_{1}^{h} G \rightarrow\left(G / P_{1}^{h} G\right) / P_{1}^{h}\left(G / P_{1}^{h} G\right) \rightarrow \ldots,
$$

which stabilizes, as a possibly transfinite tower, at a group $R$ which is $h 1$-acyclically reduced. By design, every epimorphism $G \rightarrow G^{\prime}$, with $G^{\prime} h 1$-acyclically reduced, factors through $R$. Thus $Q_{1}^{h} G=R$ is the unique maximal $h 1$-acyclically reduced quotient of $G$.

(iii) This follows from the fact that every $h$-acyclic space is also $k$-acyclic.

2.13. Remark. If $X$ is an $(n-1)$-connected space, the morphism of fibrations

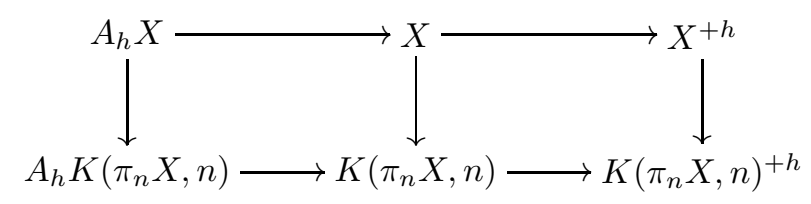

shows that, always, $\operatorname{ker}\left(\pi_{n} X \longrightarrow \pi_{n} X^{+h}\right)<P_{n}^{h} \pi_{n} X$. If $h$ is connective and $n \geq 2$, then the two groups are equal by (1.6) and (0.6). In general, we have no accurate description of the relationship between the two groups. 
If $n=1$, we know that $\operatorname{ker}\left(\pi_{1} X \rightarrow \pi_{1} X^{+h}\right)<P_{1}^{h} \pi_{1} X$ is $h 1$-perfect; combine (2.3),i and ii). Should it happen that $\operatorname{ker}\left(\pi_{1} X \rightarrow \pi_{1} X^{+h}\right)=P_{1}^{h} \pi_{1} X$, for every $X$, then $\pi_{1} X^{+h}$ depends only upon $\pi_{1} X$. Casacuberta-Rodriguez [11] call such a localizing functor $\pi_{1}$-compatible. Tai [30, 6.1] identifies the following homological plus-constructions as being $\pi_{1}$-compatible.

(i) $h_{*}(p t)$ has elements of infinite order and Bousfield's transitional dimension $d(h)$ for $h$ is $\geq 1$.

(ii) $h_{*}(p t)$ is torsion.

Bousfield's transitional dimension is defined as follows. Let $P(h)$ denote the set of primes $p$ for which $h_{*}(p t)$ is not uniquely $p$-divisible. For $p \in P(h)$ set

$$
\begin{aligned}
d_{p}(h) & :=\max \left\{n \mid K\left(\mathbb{Z}_{p}^{\wedge}, n+1\right) \text { is } h \mathbb{Z} / p \text {-local }\right\} \leq \infty \\
d(h) & :=\min \left\{d_{p}(h) \mid p \in P(h)\right\}
\end{aligned}
$$

see [7].

2.14. Corollary. If $+h$ is $\pi_{1}$-compatible, then the following hold.

(i) For every space $X, P_{1}^{h} \pi_{1}\left(X^{+h}\right)=1$.

(ii) For every group $G, P_{1}^{h}\left(G / P_{1}^{h} G\right)=1$.

2.15. Example. Let $K$ be real or complex $K$-homology. Then $d_{p}(K)=1$, for all primes $p$; see (1.8). Thus $d(K)=1$, implying that $h$ is $\pi_{1}$-compatible.

Here are some basic criteria for $\pi_{1}$-compatibility.

2.16. Lemma. For a homology theory $h$, the following are equivalent.

(i) $+h$ is $\pi_{1}$-compatible.

(ii) For every space $X, P_{1}^{h}\left(\pi_{1} X^{+h}\right)=1$.

(iii) For every group $G, P_{1}^{h}\left(G / P_{1}^{h} G\right)=1$.

(iv) For every space $X$, if $\pi_{1} X^{+h}$ is $h 1$-perfect, then $X^{+h}$ is simply connected.

(v) If $N$ and $Q$ are h1-perfect groups, then every extension of $Q$ by $N$ is $h 1$ perfect.

Proof. (i) $\Longleftrightarrow$ (ii) is (2.14).

(ii) $\Longrightarrow$ (iii) Choose $X=K(G, 1)$ in (ii).

(iii) $\Longrightarrow$ (ii) Choose a Kan-Thurston map $K(V, 1) \rightarrow X$; see [22]. It follows that $P_{1}^{h} \pi_{1} X^{+h} \cong P_{1}^{h}\left(V / P_{1}^{h} V\right)=1$.

(iii) $\Longrightarrow$ (iv) Given a space $X$ with $h 1$-perfect fundamental group, choose a Kan-Thurston map $K(U, 1) \rightarrow X$. It follows that $K(U, 1)^{+H \mathbb{Z}} \simeq X^{+H \mathbb{Z}}$ and, hence, $K(U, 1)^{+h} \simeq X^{+h}$. Thus $U / P_{1}^{h} U \cong \pi_{1} K(U, 1)^{+h} \cong \pi_{1} X^{+h}$ is a quotient of the $h 1$-perfect group $\pi_{1} X$. By (2.3), $U / P_{1}^{h} U$ is again $h 1$-perfect, hence is 1 , by hypothesis (iii).

(iv) $\Longrightarrow$ (v) Suppose $N \longmapsto G \rightarrow Q$ is an extension in which $N$ and $Q$ are $h 1$-perfect. Choose $X:=K(G, 1)$ to see that $\pi_{1} X^{+h} \cong G / P_{1}^{h} G$ is a quotient of $Q$, hence is $h 1$-perfect; see (2.3). By hypothesis (iv), $\pi_{1} X^{+h}=1$, implying that $G$ is $h 1$-perfect. 
(v) $\Longrightarrow$ (iii) A group $G$ gives rise to the following diagram of group extensions:

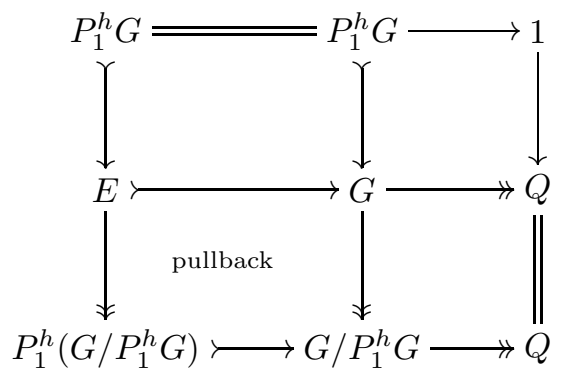

By hypothesis, $E$ is $h 1$-perfect. Moreover, $E$ contains the unique maximal $h 1$ perfect subgroup $P_{1}^{h} G$ of $G$; see 2.3 iv). Thus $E=P_{1}^{h} G$, implying that $P_{1}^{h}\left(G / P_{1}^{h} G\right)$ $=1$. This completes the proof.

Every group $G$ is the colimit of the system of its finitely generated subgroups. Here is an analogue of this fact within the category of $h n$-perfect groups.

2.17. Proposition. Every hn-perfect group $G$ is the directed colimit of the system $G_{\lambda}$ of hn-perfect subgroups such that $\operatorname{card}\left(G_{\lambda}\right)$ is less than or equal to the smallest infinite cardinal $c \geq \operatorname{card}\left(h_{*}(p t)\right)$.

Proof. We know that $G$ is a quotient of $\pi_{n} A_{h} K(G, n)$. From Bousfield [5, 11] we know that $A_{h} K(G, n)$ is the union of its acyclic subcomplexes $A_{\lambda}$ such that $A_{\lambda}$ has at most $c$ cells. Therefore each element of $\pi_{n} A_{h} K(G, n)$ belongs to some $\pi_{n} A_{\lambda}$. Further $\pi_{n} A_{\lambda}$ is generated by at most $c$ elements. Consequently, $\operatorname{card}\left(\pi_{n} A_{\lambda}\right) \leq c$, because $c$ is infinite. We find that $\pi_{n}\left(A_{h} K(G, n) \rightarrow K(G, n)\right)$ sends each $\pi_{n} A_{\lambda}$ to an $h n$-perfect subgroup $G_{\lambda}$ of $G$ with at most $c$ elements. By design, each element of $G$ belongs to some $G_{\lambda}$. The claim follows.

More generally, we make the following

2.18. Definition. A homology theory $h$ is $\pi_{n}$-compatible, $n \geq 1$, if, for every $(n-1)$-connected space $X, \pi_{n} X \rightarrow \pi_{n} X^{+h}$ depends only upon $\pi_{n} X$ (in particular, $\left.\pi_{n} X^{+h} \cong \pi_{n} K\left(\pi_{n} X, n\right)^{+h}\right)$.

By combining (0.6) with section 5 we obtain:

2.19. Proposition. Every connective $h$ is $\pi_{n}$-compatible, for $n \geq 1$.

2.20. Remark. We know of no homology theory which is not $\pi_{1}$-compatible. However, in general, homology theories need not be $\pi_{n}$-compatible if $n \geq 2$. To see this, we take $h=K$ (real or complex $K$-theory) and $X:=B \mathrm{SO}$. Now $\pi_{2} B \mathrm{SO}=\mathbb{Z} / 2$, which is $K 2$-perfect; see (1.8). On the other hand, $X$ is $K$-local by Meier's theorem [24], implying that $\operatorname{ker}\left(\pi_{2} B \mathrm{SO} \rightarrow \pi_{2} B \mathrm{SO}^{+K}\right)=0$. Thus $K$ is not $\pi_{2}$-compatible.

\section{3. $+h n$-LOCALIZATION OF ABELIAN GROUPS}

3.1. Definition. An abelian group $G$ is $+h n$-local if $K(G, n)=K(G, n)^{+h}$.

3.2. Remark. By (2.6), $G$ is $+h n$-local if and only if $\pi_{n} K(G, n) \rightarrow \pi_{n} K(G, n)^{+h}$ is an isomorphism. Therefore, if $h$ is $\pi_{n}$-compatible and $X$ is $(n-1)$-connected, then $\pi_{n} X^{+h}$ is $+h n$-local. 
We write $+h n-\mathcal{A B}$ for the full subcategory of $+h n$-local groups within $\mathcal{A B}$, the category of abelian groups. We write $\tau_{n}: \mathcal{A B} \longrightarrow+h n-\mathcal{A B}$ for the functor defined by $\pi_{n} K(G, n) \mapsto \pi_{n} K(G, n)^{+h}$. This is a localizing functor on $\mathcal{A B}$ by (2.5). From (0.2) we see that the categories $+h n-\mathcal{A B}$ form a descending tower

$$
\mathcal{A B}=:+h 0-\mathcal{A B} \supset+h 1-\mathcal{A B} \supset \ldots
$$

3.3. Corollary. For all abelian groups $G$ and $n \geq 1, \tau_{n} G=\tau_{n}\left(\tau_{n-1} G\right)$.

The following examples illustrate the effect of the functor $\tau_{n}$. The proofs follow directly from the works of Bousfield [6] and Mislin [26].

3.4. Example. Here $h=H(-; \mathbb{Z} / p)$ for a prime $p$. If $n \geq 2$, then $\tau_{n}$ is the group $\operatorname{Ext}\left(\mathbb{Z}_{p^{\infty}}, G\right)$, the "ext-p-completion" of the abelian group $G$. The maximal $h 1$ perfect subgroup of $G$ is $P_{1}^{h} G=\bigcup\{U<G \mid U / p U=1\}$. Thus $\tau_{1} G=G / P_{1}^{h} G=$ $\operatorname{im}\left(G \rightarrow \operatorname{Ext}\left(\mathbb{Z}_{p \infty}, G\right)\right)$.

3.5. Example. Here $h=K$, real or complex $K$-theory. For an abelian group $G$ we have $\tau_{1} G=G, \tau_{2} G=G / \operatorname{torsion}(G)$ and $\tau_{n} G=\mathbb{Q} \otimes_{\mathbb{Z}} G$ if $n \geq 3$.

3.6. Lemma. For $n \geq 1$, let $\varphi: L_{1} \rightarrow L_{2}$ be a map between + hn-local abelian groups. Then $\operatorname{ker}(\varphi)$ is + hn-local and $\operatorname{coker}(\varphi)$ is $+h(n-1)$-local.

Proof. By (7.2), we have $\operatorname{fib} K(\varphi, n) \simeq K(\operatorname{coker}(\varphi), n-1) \times K(\operatorname{ker}(\varphi), n)$ and is $+h n$-local. The result follows.

3.7. Theorem. Let $n \geq 1$ and let $G$ be a group, abelian if $n \geq 2$. Then the group $K\left(P_{n}^{h} G, n\right)^{+h}$ is the $n$-connected cover of $K(G, n)^{+h}$.

Proof. For $n=1$, this follows from the definition of $P_{1}^{h} G$ and lemma (0.3). For $n>1$, it suffices to show that $K\left(P_{n}^{h} G, n\right)^{+h} \rightarrow K(G, n)^{+h}$ induces an isomorphism in $\pi_{n+1}$; see (2.5). Indeed, using the notation of lemma (2.5), we have an exact sequence

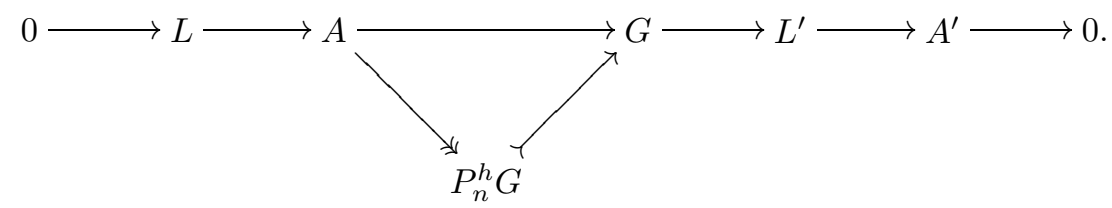

By classifying the short exact sequence on the left we obtain the fibration $K(A, n) \rightarrow$ $K\left(P_{n}^{h} G, n\right) \longrightarrow K(L, n+1)$. Apply $+h$-localization to obtain the fibration $* \simeq$ $K(A, n)^{+h} \rightarrow K\left(P_{n}^{h} G, n\right)^{+h} \rightarrow K(L, n+1)$, from which the claim follows.

\section{4. $h n$-CENTRAL EXTENSIONS}

Given a homology theory $h$ and a group $G$, according to (7.7), we have the central extension $\pi_{n+1} K(G, n)^{+h} \longmapsto \pi_{n} A_{h} K(G, n) \rightarrow P_{n}^{h} G$. In addition, this sequence inherits universal properties from the universal properties of the colocalization/ localization-fibration $A_{h} K(G, n) \rightarrow K(G, n) \rightarrow K(G, n)^{+h}$.

4.1. Definition. A central extension of groups $L \longmapsto H \rightarrow G$ is called $h n$-central if $L$ is $+h(n+1)$-local. 
4.2. Definition. A central extension

$$
A \longmapsto E \longrightarrow G
$$

is universal with respect to $h n$-central extensions if every diagram

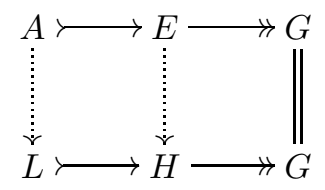

with $h n$-central bottom row, can be filled uniquely as indicated. If, in addition, $A$ is $+h(n+1)$-local, then we call $(C)$ a universal $h n$-central extension.

The main objective of this section is to establish the following statements.

4.3. Theorem. Every hn-perfect group $G, n \geq 1$, gives rise to the central extension

$$
\pi_{n+1} K(G, n)^{+h} \longmapsto \pi_{n} A_{h} K(G, n) \longrightarrow G
$$

which is universal with respect to hn-central extensions. Moreover, $(C)$ is a universal hn-central extension if $G$ is abelian or if $h$ is $\pi_{2}$-compatible; see (2.18).

4.4. Proposition. Suppose $G$ is abelian or $h$ is $\pi_{2}$-compatible. Then, for $n \geq 1$, the $+h(n+1)$-local group $\pi_{n+1} K(G, n)^{+h}$ is a representing object for the functor

$$
\operatorname{Ext}\left(P_{n}^{h} G,-\right)=H^{2}\left(P_{n}^{h} G ;-\right):+h(n+1)-\mathcal{A B} \longrightarrow \mathcal{A B} .
$$

Proof. This follows from (4.12).

4.5. Remark. This is very much like in the classical situation considered by Kervaire: For any group $G, \pi_{2} K(G, 1)^{+}$is the representing object for the functor on $\mathbb{Z}$-modules

$$
H^{2}(P G ;-) \cong \operatorname{Hom}\left(H_{2}(P G ; \mathbb{Z}),-\right) \cong \operatorname{Hom}\left(\pi_{2} K(G, 1)^{+},-\right),
$$

where $P G$ denotes the maximal perfect subgroup of $G$.

4.6. Theorem. Suppose $h$ is $\pi_{2}$-compatible. If $G$ is an arbitrary group, then

$$
\pi_{3} K(G, 1)^{+h} \cong H_{3}\left(K\left(\widetilde{P_{1}^{h} G}, 1\right)^{+h} ; \mathbb{Z}\right)
$$

where $\widetilde{P_{1}^{h} G}$ is the universal h1-central extension of $P_{1}^{h} G$; see (4.3).

Finally, we analyze the situation where $n=1$ and $h$ is not necessarily $\pi_{2}$ compatible. For an arbitrary abelian group $H$ we have the natural isomorphism $\operatorname{Hom}\left(\tau_{2} H, L\right) \cong \operatorname{Hom}(H, L)$ whenever $L$ is $+h 2$-local. Moreover, if $G$ is any $h 1$ perfect group, then $H_{1} G$ is $h 1$-perfect too and, therefore, $K\left(H_{1} G, 1\right)^{+h} \cong K(\Lambda, 2)$ for some $+h 2$-local group $\Lambda$. Thus, for an arbitrary group $G$,

$$
\tau_{2} H_{2}\left(H_{1} P_{1}^{h} G ; \mathbb{Z}\right)=H_{2}\left(H_{1} P_{1}^{h} G ; \mathbb{Z}\right) .
$$

As a result, we can compute the image of $\pi_{2} K(G, 1)^{+h} \cong \pi_{2} K\left(P_{1}^{h} G, 1\right)^{+h}$ under the functor $\tau_{2}$ as follows. 
4.7. Proposition. Associated to an h1-perfect group $G$ there is the natural short exact sequence

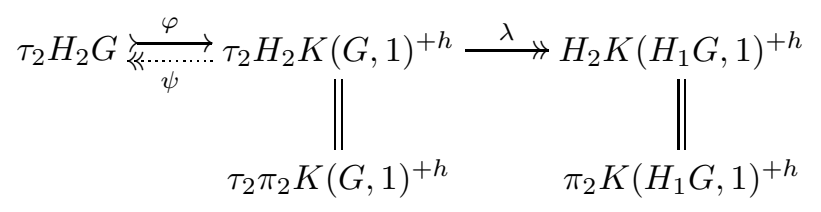

The sequence splits, but not naturally. Moreover, $\tau_{2} \pi_{2} K(G, 1)^{+h}$ is the kernel of the universal h1-central extension of $G$.

We proceed to prove these statements and establish some related facts.

Proof of theorem (4.3). From (7.7) we know that $(C)$ is a central extension. To establish its universal property, let $(\zeta): L \succ H \rightarrow G$ be an $h n$-central extension. We need to show that the identity map on $G$ lifts to a unique morphism $\beta: \pi_{n} A_{h} K(G, n) \rightarrow H$. To see that $\beta$ exists, consider the commutative diagram below:

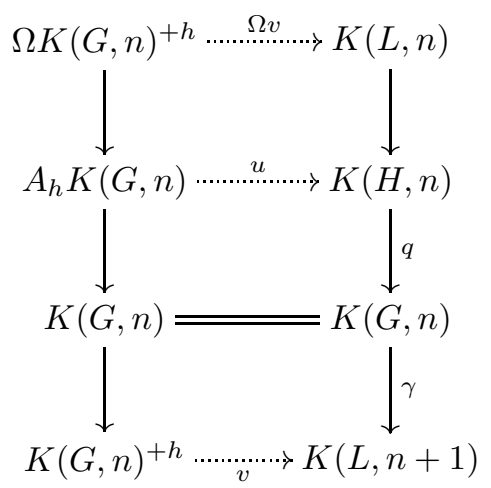

Here $\gamma$ classifies the principal fibration associated to $(\zeta)$. The universal property of the localizing map $K(G, n) \rightarrow K(G, n)^{+h}$ yields $v$ uniquely such that the bottom square commutes. Thus a map $u$ exists, and $\pi_{n} u$ is a candidate for $\beta$. To see that $\beta$ is unique, we argue as follows. Suppose $\beta^{\prime}: \pi_{n} A_{h} K(G, n) \rightarrow H$ also makes the diagram commute. Let $\bar{\beta}$ denote the function obtained by following $\beta$ by the group inverse operation on $H$. Then $\beta^{\prime} \bar{\beta}: \pi_{n} A_{h} K(G, n) \rightarrow H$ is a homomorphism because $L$ is central in $H$. Realize $\beta^{\prime} \bar{\beta}$ by a map $u^{\prime} \bar{u}: A_{h} K(G, n) \rightarrow K(H, n)$. This is possible since $A_{h} K(G, n)$ is $(n-1)$-connected; see (2.8) if $n \geq 2$. By design, $q \circ\left(u^{\prime} \bar{u}\right) \simeq 0$. Consequently, $u^{\prime} \bar{u}: A_{h} K(G, n) \longrightarrow K(L, n)$, which is 0 because $A_{h} K(G, n)$ is $h$-acyclic and $K(L, n)$ is $h$-local. Thus $\beta=\beta^{\prime}$.

Finally, $\pi_{n+1} K(G, n)^{+h}$ is $+h(n+1)$-local if $G$ is abelian, see (2.8), or if $h$ is $\pi_{2}$-compatible; see (2.18). The proof is complete.

4.8. Lemma. If $h$ is $\pi_{2}$-compatible and $G$ is $h 1$-perfect, then $K\left(\pi_{1} A_{h} K(G, 1), 1\right)^{+h}$ is 2-connected.

Proof. Write $U:=\pi_{1} A_{h} K(G, 1)$, the universal $h 1$-central extension of $G$; see (4.3). The cofiber sequence $A_{h} K(G, 1) \rightarrow K(U, 1) \rightarrow \Gamma$ has $\Gamma$ 1-connected and induces in $H(-; \mathbb{Z})$ the exact sequence

$$
H_{2} A_{h} K(G, 1) \longrightarrow H_{2} K(U, 1) \longrightarrow H_{2} \Gamma \longrightarrow H_{1} A_{h} K(G, 1) \stackrel{\cong}{\longrightarrow} H_{1} K(U, 1) .
$$


Thus $\Gamma$ is 2-connected. But $K(U, 1) \rightarrow \Gamma$ is a $+h$-equivalence, because $A_{h} K(G, 1) \rightarrow$ $*$ is. Consequently, we get a homotopy equivalence $K(U, 1)^{+h} \longrightarrow \Gamma^{+h}$, with $\Gamma^{+h}$ 2-connected, since $h$ is $\pi_{2}$-compatible.

As a corollary, we obtain the

Proof of theorem (4.6). We know from (3.7) that $\pi_{3} K(G, 1)^{+h} \cong \pi_{3} K\left(P_{1}^{h} G, 1\right)^{+h}$. The universal $h 1$-central extension $C \longmapsto U \rightarrow P_{1}^{h} G$ yields the framed fibration

$$
K(C, 1) \rightarrow K(U, 1) \rightarrow K\left(P_{1}^{h} G, 1\right) \stackrel{\gamma}{\rightarrow} K(C, 2),
$$

which is classified by $\gamma . K(C, 2)$ is $+h$-local. Thus we obtain the fibration

$$
K(U, 1)^{+h} \rightarrow K\left(P_{1}^{h} G, 1\right)^{+h} \rightarrow K(C, 2) ;
$$

see [18, p. 74]. In the long exact sequence of homotopy groups of this fibration, we find the isomorphism $\pi_{3} K(U, 1)^{+h} \rightarrow \pi_{3} K\left(P_{1}^{h} G, 1\right)^{+h}$. The first group is isomorphic to $H_{3} K(U, 1)^{+h}$, by (4.8). This completes the proof.

4.9. Lemma. Let $(\zeta): L \longmapsto U \rightarrow G$ be a universal hn-central extension, and let $\left(\zeta^{\prime}\right): L^{\prime} \longmapsto H \rightarrow G$ be an hn-central extension. If $H$ is hn-perfect, then the unique map $\beta: U \rightarrow H$ is onto.

Proof. The universal property of $(\zeta)$ yields uniquely the commutative diagram below:

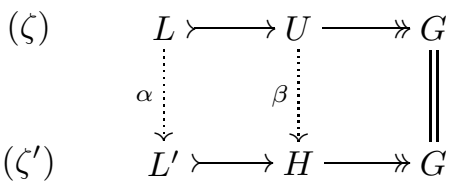

An arbitrary element of $H$ differs from $\operatorname{im}(\beta)$ by an element of $L$, implying that $\operatorname{im}(\beta)$ is normal in $H$. Therefore, $\operatorname{coker}(\alpha) \cong \operatorname{coker}(\beta)$. But $\operatorname{coker}(\beta)$ is $h n$-perfect by $(2.3 \mathrm{i})$, and coker $(\alpha)$ is $h n$-local by (3.6). Thus both groups are trivial, implying that $\beta$ is onto.

The following facts are needed in order to bring methods from homological algebra to bear on $h n$-central extensions.

4.10. Lemma. If $G$ is an abelian hn-perfect group, $n \geq 1$, then every hn-central extension of $G$ is abelian.

Proof. For a central extension $L \longmapsto E \rightarrow G$, the commutator map $\gamma: E \times E \longrightarrow E$, $\gamma(t, x):=t x t^{-1} x^{-1}$, factors to a bilinear map $\widehat{\gamma}: G \times G \rightarrow L$. Now $\widehat{\gamma}$ is trivial because $G \times G$ is $h n$-perfect, (2.3)i , and $L$ is $h n$-local; see (0.2). The claim follows.

4.11. Proposition. To an h1-perfect group $G$, there is associated the universal h1-central extension $(\zeta G)$, given by the pushout construction below:

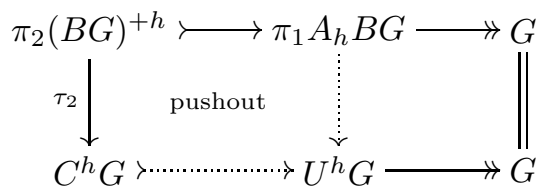

Here $C^{h} G:=\tau_{2}\left(\pi_{2} K(G, 1)^{+h}\right)$; see section 3 . 
Proof. $(\zeta G)$ is $h 1$-central by design. To establish its universal property, consider the commutative diagram.

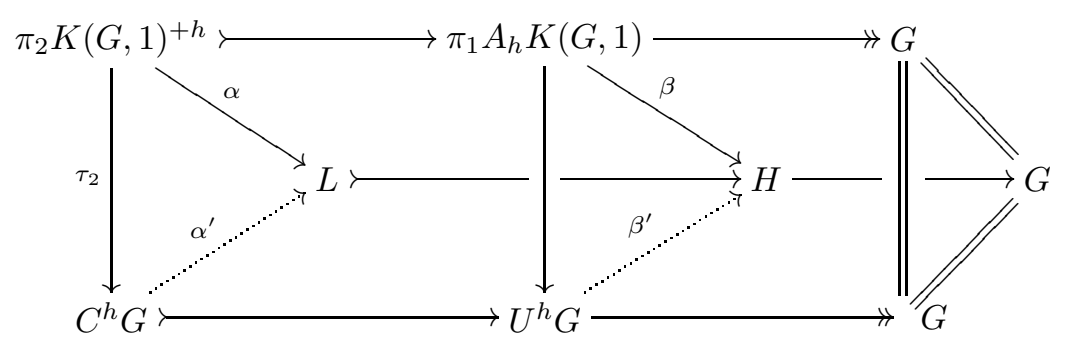

$(\zeta)$ is $h n$-central. Therefore we are entitled to the unique morphism $(C G) \rightarrow(\zeta)$, by (4.3). The unique map $\alpha^{\prime}$ comes from the universal property of the localizing map $\tau_{2}$. The unique map $\beta^{\prime}$ over $\operatorname{Id}_{G}$ comes from the pushout construction of $U^{h} G$. This completes the proof.

4.12. Corollary. Given an abelian + hn-perfect group $G, n \geq 1$, let $(\zeta G): L \longmapsto$ $E \rightarrow G$ be the universal hn-central extension of $G$; see (4.3). If $L^{\prime}$ is an arbitrary $+h(n+1)$-local group, then there is the natural equivalence $\operatorname{Hom}\left(L, L^{\prime}\right) \rightarrow$ $\operatorname{Ext}\left(G, L^{\prime}\right)$.

4.13. Proposition. For an hn-perfect group $G$ and $a+h(n+1)$-local group $L$, there is a natural short exact sequence

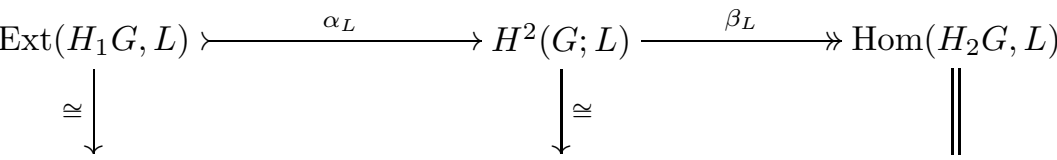

$\operatorname{Hom}\left(\pi_{n+1} K\left(H_{1} G, n\right)^{+h}, L\right) \longleftrightarrow \operatorname{Hom}\left(\pi_{n+1} K(G, n)^{+h}, L\right) \longrightarrow \operatorname{Hom}\left(H_{2} G, L\right)$

Moreover, $\alpha_{L}$ is an isomorphism whenever $G$ is abelian.

Proof. The top row is the universal coefficient sequence. $H^{2}(G ; L)$ classifies central extensions of $G$ by $L$. Such extensions are in natural bijective correspondence with $\operatorname{Hom}\left(\pi_{n+1} K(G, n)^{+h}, L\right)$, using (4.3). Further, $\operatorname{Ext}\left(H_{1} G, L\right)$ consists of abelian extensions of the $h 1$-perfect group $H_{1} G$ by $L$. According to (4.10), all central extensions of $H_{1} G$ by $L$ are automatically abelian. The map

$$
\operatorname{Hom}\left(\pi_{n+1} K\left(H_{1} G, n\right)^{+h}, L\right) \rightarrow \operatorname{Ext}\left(H_{1} G, L\right)
$$

is a natural isomorphism by (4.3). The claim follows.

4.14. Corollary. If $G$ is abelian, then $\operatorname{Hom}\left(H_{2} G, L\right)=0$, whenever $G$ is hnperfect and $L$ is $+h(n+1)$-local.

4.15. Corollary. If $G$ is abelian and hn-perfect, then $H_{2}(G ; \mathbb{Z})$ is $h(n+1)$-perfect.

Proof. From (4.14) we see that $\operatorname{Hom}\left(H_{2} G, L\right)=0$, whenever $L$ is $+h(n+1)$-local. But

$$
\begin{aligned}
\operatorname{Hom}\left(H_{2} G, L\right) & =\left[K\left(H_{2} G, n+1\right), K(L, n+1)\right] \\
& =\left[K\left(H_{2} G, n+1\right)^{+h}, K(L, n+1)\right]
\end{aligned}
$$

Choosing $L:=\pi_{n+1} K\left(H_{2} G, n+1\right)^{+h}$ shows that $H_{2} G$ is $+h(n+1)$-perfect. 
Proof of (4.7). From (4.13) we get, for each $+h 2$-local $L$, the natural short exact sequence

$$
\operatorname{Hom}\left(H_{2} K\left(H_{1} G, 1\right)^{+h}, L\right) \stackrel{\alpha_{L}}{\longrightarrow} \operatorname{Hom}\left(\tau_{2} H_{2} K(G, 1)^{+h}, L\right) \stackrel{\beta_{L}}{\longrightarrow} \operatorname{Hom}\left(\tau_{2} H_{2} G, L\right) .
$$

This yields the split short exact sequence in question for formal reasons; see (6.1). The identification of $\tau_{2} \pi_{2} K(G, 1)^{+h}$ as the kernel of the universal $h 1$-central extension of $G$ follows from (4.11).

\section{EXAMPLES}

Here we consider the two cases where $h=H\left(-, \mathbb{Z}_{P}\right), P$ a set of primes, or $h=H(-, \mathbb{Z} / p), p$ a prime.

5.1. Example. Let $h=H\left(-; \mathbb{Z}_{P}\right)$. In this case, each Moore space $M(\mathbb{Z} / p, 1)$, $p \notin P$, is $h$-acyclic. Thus $+h$-localization factors through Anderson's localization; see [1], compare [10]. Consequently a $+h$-local space $X$ has higher homotopy groups which are $\mathbb{Z}_{P}$-modules and, for $p \notin P$, the $p$-th power function on $\pi_{1} X$ is injective. If $X$ is simply connected then $X$ is $+h$-local if and only if $X$ is $h$-local, which holds if and only if all homotopy groups of $X$ are $\mathbb{Z}_{P}$-modules; compare (1.6). In particular, $h$ is $\pi_{n}$-compatible, for all $n \geq 1$. Also, a group is $h 1$-perfect if and only if $H_{1}\left(G ; \mathbb{Z}_{P}\right)=0$. Generalizing Kervaire's results, see [23], compare [3] Chap. 8], we conclude at the level of fundamental groups that:

(i) For every group $G, P_{1}^{h} \pi_{1} K(G, 1)^{+h}=1$; i.e. $h$ is $\pi_{1}$-compatible.

(ii) $\pi_{2} K(G, 1)^{+h} \longmapsto \pi_{1} A_{h} K(G, 1) \rightarrow P_{1}^{h} G$ is the universal $h 1$-central extension of $P_{1}^{h} G$.

(iii) In view of the discussion preceding (4.7), we have

$$
\pi_{2} K(G, 1)^{+h} \cong \pi_{2} K\left(P_{1}^{h} G, 1\right)^{+h} \cong H_{2}\left(K\left(P_{1}^{h} G, 1\right)^{+h} ; \mathbb{Z}\right) .
$$

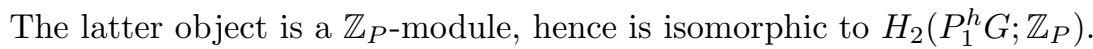

(iv) $\pi_{3} K(G, 1)^{+h} \cong H_{3}\left(K\left(\widetilde{P_{1}^{h} G}, 1\right)^{+h} ; \mathbb{Z}\right) \cong H_{3}\left(\widetilde{P_{1}^{h} G} ; \mathbb{Z}_{P}\right)$, where $\widetilde{P_{1}^{h} G}$ denotes the universal $h 1$-central extension of $P_{1}^{h} G$; see (4.6).

At the level of higher Eilenberg-MacLane spaces we conclude that $K(G, n)$ is $+h n$ perfect if and only if $G$ is $P^{\prime}$ torsion. In this case $K(G, n)$ is $h$-acyclic. For an arbitrary abelian group $G$, the $+h$-construction yields the fibration

$$
K\left(T_{P^{\prime}} G, n\right) \times K\left(\operatorname{coker}\left(G \rightarrow \mathbb{Z}_{P} \otimes G\right), n-1\right) \rightarrow K(G, n) \longrightarrow K\left(\mathbb{Z}_{P} \otimes G, n\right),
$$

where $T_{P^{\prime}} G$ denotes the $P^{\prime}$-torsion subgroup of $G$.

5.2. Example. Let $h=H(-; \mathbb{Z} / p)$, where $p$ is a prime. On simply connected spaces we know that $+h$-localization agrees with $h$-localization; see (1.7). Further, on $p$-good spaces, $h$-localization agrees with $p$-completion in the sense of BousfieldKan [9].

Thus, for an abelian group $G$ and $n \geq 2$, [5, 4.3] yields the short exact sequence

$$
1 \longrightarrow \operatorname{Ext}\left(\mathbb{Z}_{p^{\infty}}, \pi_{i} K(G, n)\right) \longleftrightarrow \pi_{i} K(G, n)^{+h} \longrightarrow \operatorname{Hom}\left(\mathbb{Z}_{p^{\infty}}, \pi_{i-1} K(G, n)\right) \text {. }
$$

Therefore $G$ is $h n$-perfect if and only if $\operatorname{Ext}\left(\mathbb{Z}_{p^{\infty}}, G\right)=0$. According to [9, p. 166], this happens exactly when $\lim ^{1}\left\{\operatorname{Hom}\left(\mathbb{Z} / p^{n}, G\right)\right\}=0=\lim \left\{G / p^{k} G\right\}$.

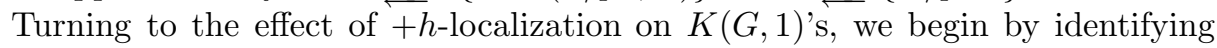
$h 1$-perfect groups. 
5.3. Lemma. For $h=H(-; \mathbb{Z} / p)$, a group $G$ is $h 1$-perfect if and only if $H_{1}(G ; \mathbb{Z} / p)$ $=0$.

Proof. If $G$ is $h 1$-perfect, then $K(G, 1)^{+h}$ is 1 -connected. Thus $H_{1}(G ; \mathbb{Z} / p)=0$ because $K(G, 1) \rightarrow K(G, 1)^{+h}$ induces an $h$-isomorphism. Conversely, if $H_{1}(G ; \mathbb{Z} / p)$ $=0$, then $K(G, 1)^{h}$ is 1 -connected. To see this, recall [6] that $\pi_{1} K(G, 1)^{h}$ is an $h$-local group, and such a group $\Gamma$ vanishes exactly when $H_{1}(\Gamma ; \mathbb{Z} / p)=0$. By (1.6), $K(G, 1)^{h} \simeq K(G, 1)^{+h}$, implying that $G$ is $h 1$-perfect.

We know from $(2.3 \mathrm{v})$ that every group $G$ has a unique maximal $H \mathbb{Z} / p$-perfect subgroup $P_{1}^{h} G$; i.e. $P_{1}^{h} G$ is maximal in $G$ with $\mathbb{Z} / p \otimes H_{1}\left(P_{1}^{h} G ; \mathbb{Z}\right)=0$. $H \mathbb{Z} / p$ is seen to be $\pi_{1}$-compatible, either by [30,6.1], or by showing that $h 1$-perfect groups are closed under extensions (Serre spectral sequence) and invoking (2.16). Also, if $X$ is $(n-1)$-connected and $n \geq 2$, then (cf. Bousfield [5, 4.3]) $\pi_{n} X \rightarrow \pi_{n} X^{h}$ agrees with $\pi_{n} X \rightarrow \operatorname{Ext}\left(\mathbb{Z}_{p^{\infty}}, \pi_{n} X\right)$ and $h$ is thus $\pi_{n}$-compatible for $n \geq 2$ as well. This, in conjunction with the discussion in (5.1), extablishes the claim (2.19).

5.4. Proposition. For $h=H(-; \mathbb{Z} / p)$, the group $\pi_{2} K(G, 1)^{+h}$ fits into the natural short exact sequence

$$
\operatorname{Ext}\left(\mathbb{Z}_{p^{\infty}}, H_{2}\left(P_{1}^{h} G ; \mathbb{Z}\right)\right) \longleftrightarrow \pi_{2} K(G, 1)^{+h} \longrightarrow \operatorname{Hom}\left(\mathbb{Z}_{p^{\infty}}, H_{1}\left(P_{1}^{h} G ; \mathbb{Z}\right)\right) .
$$

The sequence splits, but not naturally.

Proof. From (4.7) we get the natural short exact sequence

$$
\tau_{2} H_{2}\left(P_{1}^{h} G ; \mathbb{Z}\right) \longmapsto \pi_{2} K(G, 1)^{+h} \rightarrow \pi_{2} K\left(H_{1}\left(P_{1}^{h} G ; \mathbb{Z}\right), 1\right)^{+h},
$$

which splits. The terms at the end are homotopy groups of $h$-localizations of abelian Eilenberg-MacLane spaces. Further, [9, p. 183],

$$
\tau_{2} H_{2}\left(P_{1}^{h} G ; \mathbb{Z}\right) \cong \operatorname{Ext}\left(\mathbb{Z}_{p^{\infty}}, H_{2}\left(P_{1}^{h} G ; \mathbb{Z}\right)\right)
$$

and

$$
\pi_{2} K\left(H_{1} P_{1}^{h} G, 1\right)^{+h} \cong \operatorname{Hom}\left(\mathbb{Z}_{p^{\infty}}, H_{1}\left(P_{1}^{h} G ; \mathbb{Z}\right)\right) .
$$

\section{An ALGEBRAiC LEMMA}

Let $\mathcal{C}$ be a class of abelian groups with the following properties:

(i) $\mathcal{C}$ is closed under products.

(ii) If $A \in \mathcal{C}$ and $A \cong B \times C$, then $B, C \in \mathcal{C}$.

The sole purpose of this section is to formulate the following lemma, which we need in the proof of (4.7).

6.1. Lemma. Suppose for groups $A, B, C \in \mathcal{C}$ there is, for each $L \in \mathcal{C}$, a natural short exact sequence

(Hom-S)

$$
\operatorname{Hom}(A, L) \stackrel{\alpha_{L}}{\longrightarrow} \operatorname{Hom}(B, L) \stackrel{\beta_{L}}{\longrightarrow} \operatorname{Hom}(C, L) .
$$

Then there is a natural short exact sequence

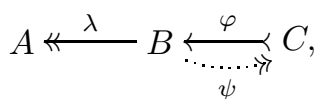

which induces (Hom-S). Further, (S) splits, but not naturally. 
Proof. We obtain maps $\lambda, \phi$ and $\psi$ as follows: Choose $L:=B$ to find the map $\beta_{B}\left(\operatorname{Id}_{B}\right)=: \varphi: C \rightarrow B$. It induces $\beta_{L}$ for arbitrary $L$. Next choose $L:=C$. Since $\beta_{L}$ is onto, there exists some $\psi: B \rightarrow C$ such that $\beta_{L}(\psi)=\operatorname{Id}_{C}$. It follows that $\psi \circ \varphi=\operatorname{Id}_{C}$ and, therefore, $\phi$ is a split monomorphism and $\psi$ is onto. Next choose $L:=A$ to find the map $\lambda:=\alpha_{A}\left(\operatorname{Id}_{A}\right): B \longrightarrow A$. The proof that these maps satisfy the required properties is a bit tedious but entirely elementary. We omit it.

\section{SOME FIBER LEMMAS}

The lemma and its corollary below are presumably known, even though we are unable to find a reference. They are included here because the corollary is needed in the proof of (3.6).

7.1. Lemma. Given a based continuous map $f: E \rightarrow B$, let $E^{\prime}=\{(e, \alpha) \in$ $\left.E \times B^{I} \mid f(e)=\alpha(0)\right\}$ and let $f^{\prime}: E^{\prime} \rightarrow B,(e, \alpha) \mapsto \alpha(1)$, be the homotopy theoretical replacement of $f$ by the fibration $f^{\prime}$. Then the following hold. If $f$ is a homomorphism of topological monoids (topological groups, abelian topological groups), then $\mathrm{fib}(f)$ is a topological submonoid (topological subgroup, abelian topological subgroup) of $E^{\prime}$.

Proof. Since $E$ and $B$ are topological monoids (topological groups, topological abelian groups), so is $E^{\prime}$ via the operation $E^{\prime} \times E^{\prime} \longrightarrow E^{\prime},(e, \alpha) \cdot\left(e_{1}, \alpha_{1}\right)=$ $\left(e e_{1}, \alpha \alpha_{1}\right)$, and from this the claim can be read.

7.2. Corollary. Let $f: K(A, n) \rightarrow K(B, n)$ be a continuous map between abelian Eilenberg-MacLane spaces, $n \geq 1$. Then

$$
\operatorname{fib}(f) \simeq K\left(\operatorname{ker}\left(\pi_{n} f\right), n\right) \times K\left(\operatorname{coker}\left(\pi_{n} f\right), n-1\right) .
$$

Proof. We may assume that $f$ is a homomorphism of abelian topological groups. By (7.1), $\mathrm{fib}(f)$ is an abelian topological group. Thus $\mathrm{fib}(f)$ is a retract of the infinite symmetric product $\mathrm{SP}^{\infty} \mathrm{fib}(f)$ and, hence, is a product of Eilenberg-MacLane spaces

$$
\mathrm{fib}(f) \simeq \prod_{k \geq 1} K\left(\pi_{k} \mathrm{fib}(f), k\right) ;
$$

see [13, compare [14, p. 88f]. The claim follows.

The proposition below is the key to all centrality phenomena which are associated with fibrations of the form $A_{h} X \longrightarrow X \longrightarrow X^{+h}$. It constitutes a strengthened version of [20, A.2].

7.3. Proposition. Let $f: E \rightarrow B$ be a morphism of topological monoids which have a homotopy inverse. Then $\Omega B \stackrel{j}{\longrightarrow} \mathrm{fib}(f) \longrightarrow E$ is a homotopy central extension; i.e. the commutator map

$$
\gamma: \Omega B \times \mathrm{fib}(f) \stackrel{j \times \operatorname{Id}}{\longrightarrow} \mathrm{fib}(f) \times \mathrm{fib}(f) \stackrel{[-,-]}{\longrightarrow} \mathrm{fib}(f)
$$

is null homotopic.

It is possible to give a direct proof of this claim. It follows, in spirit, the classical argument that higher homotopy groups are commutative; see e.g. [33, p.125]. An alternate argument can be based on unpublished work of M. Arkowitz [2]. However, for our present purposes, it will suffice to establish the following corollary (77.7) which only depends upon the much simpler self-contained development below. 
7.4. Definition. A based fibration $F \stackrel{i}{\rightarrow} E \rightarrow X$ is called $\Pi$-central if all Whitehead products $\left[i_{*} \alpha, \beta\right]$ vanish, where $\alpha \in \pi_{p} F, \beta \in \pi_{q} E$ and $p, q \geq 1$.

7.5. Example. The path fibration over any based space $X$ is $\Pi$-central.

7.6. Lemma. Any pullback of a П-central fibration along a based map is П-central.

Proof. Given a pullback diagram of a П-central fibration,

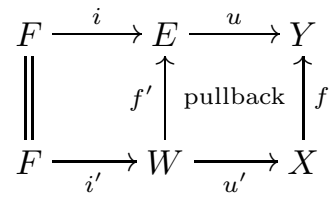

we need to check that $\left[i_{*}^{\prime} \alpha, \beta\right]=0$ whenever $\alpha \in \pi_{p} F$ and $\beta \in \pi_{q} W$. Writing $W$ as the appropriate subspace of $E \times X$, we find that $i_{*}^{\prime} \alpha=\left(f^{\prime} i^{\prime} \alpha, u^{\prime} i^{\prime} \alpha\right)=(i \alpha, 0)$ and $\beta=\left(f^{\prime} \beta, u^{\prime} \beta\right)$. Therefore, $\left[i_{*} \alpha, \beta\right]=\left(\left[i \alpha, f^{\prime} \beta\right], 0\right)=(0,0)$, since $F \rightarrow E \rightarrow Y$ is a П-central fibration.

7.7. Corollary. If $f: Y \rightarrow X$ is a based map, then $\Omega X \rightarrow \operatorname{fib}(f) \rightarrow Y$ is a П-central fibration.

\section{REFERENCES}

1. Donald W. Anderson, Localizing CW complexes, Ill. J. Math. 16 (1972), 519-525. MR 45:4401

2. M. Arkowitz, The loop of a homotopy action, unpublished, 1-7.

3. A.J. Berrick, An approach to algebraic K-theory, Res. Notes Math., no. 56, Pitman, Boston, MA-London, 1982. MR 84g:18028

4. A.K. Bousfield, Types of acyclicity, J. Pure Appl. Algebra 4 (1974), 293-298. MR 51:4220

5. - The localization of spaces with respect to homology, Topology 14 (1975), 133-150. MR 52:1676

6. Homological localization towers for groups and $\Pi$-modules, AMS Memoirs, no. 186, Amer. Math. Soc., Providence, RI, 1977. MR 56:5688

7. 104 (1982), no. 5, 1025-1029. MR 84g:55014

8. - Homotopical localizations of spaces, American J. Math. 119 (1997), no. 6, 1321-1354. MR 98m:55009

9. A.K. Bousfield and D.M. Kan, Homotopy limits, completions and localizations, second corrected printing ed., Lect. N. Math., vol. 304, Springer-Verlag, Berlin/New York, 1987. MR 51:1825 (1st ed.)

10. C. Casacuberta, Anderson localization from a modern point of view, Contemp. Math. 181 (1995), 35-44. MR 86a:55017

11. C. Casacuberta and J.L. Rodriguez, On towers approximating homological localizations, J. London Math. Soc. 56 (1997), 645-656. MR 99b:15016

12. C. Casacuberta, J.L. Rodriguez, and J.Y. Tai, Localizations of abelian Eilenberg-Mac Lane spaces of finite type, preprint (1997).

13. Albrecht Dold and René Thom, Quasifaserungen und unendliche symmetrische produkte, Annals Math. 67 (1958), 239-281. MR 20:3542

14. E. Dror Farjoun, Higher homotopies of natural constructions, J. Pure Appl. Algebra 108 (1996), no. 1, 23-34. MR 97c:55020]

15. E. Dror Farjoun and J.H. Smith, Homotopy localization nearly preserves fibrations, Topology 34 (1994), no. 2, 359-375. MR 96c:55014

16. Emmanuel Dror Farjoun, Homotopy localization and vi-periodic spaces, Algebraic Topology Homotopy and Group Cohomology (Berlin New York) (J. Aguadé, M. Castellet, and F.R. Cohen, eds.), Lect. Notes in Math., no. 1509, Springer-Verlag, 1992, pp. 104-114. MR 93k:55013

17. _ Localizations, fibrations, and conic structures, preprint (1992). 
18. - Cellular Spaces, Null Spaces, and Homotopy Localization, Lect. Notes in Math., no. 1622, Springer-Verlag, Berlin New York, 1995. MR 98f:55010

19. W.G. Dwyer and D.M. Kan, The enveloping ring of a П-algebra, Advances in Homotopy Theory (Cambridge), vol. 139, Cambridge UP, 1989, pp. 49-60. MR 91j:55015

20. J.C. Hausmann and D. Husemoller, Acyclic maps, L'Enseignement Mathématique 25 (1979), 53-75. MR 80k:55044

21. Philip S. Hirschhorn, Localization, cellularization, and homotopy colimits, Book draft, 1995.

22. Daniel M. Kan and William P. Thurston, Every connected space has the homology of a $K(\pi, 1)$, Topology 15 (1976), no. 3, 253-258. MR 54:1210

23. Michel A. Kervaire, Multiplicateurs de Schur et K-théorie, Essays on Topology and related topics: Mémoires dédiés à Georges de Rham (1903- ) (Berlin New York) (A. Haefliger and R. Narasimhan, eds.), Springer-Verlag, 1970, pp. 212-225. MR 43:321]

24. Willi Meier, Acyclic maps and knot complements, Math. Annalen 243 (1979), no. 3, 247-259. MR 81i:55020

25. _ Pullback theorems and phantom maps, Quart. J. Math., Ox. Ser. 229 (1979), 469481. MR 80g:55023

26. G. Mislin, Localization with respect to K-theory, J. Pure and Applied Algebra 10 (1977), 201-213. MR 57:7585

27. Daniel G. Quillen, Cohomology of groups, Actes du Congrès International des Mathématiciens (Nice, 1970) (Paris), vol. 2, Comité d'Organisation du Congrès, Gauthier Villars, 1971, pp. 47-51. MR 58:7627a

28. Higher $K$-theory for categories with exact sequences, New Developments in Topology (Proc. Sympos. Algebraic Topology, Oxford, 1972) (Cambridge, UK) (G.B. Segal, ed.), Lond. Math. Soc. Lec. Notes Ser., no. 11, Cambridge Univ. Press, 1974, pp. 95-103. MR 49:384

29. Jin-Yen Tai, On generalized plus-constructions of Eilenberg-Mac Lane spaces, preprint (1997), $1-14$.

30. Generalized plus-constructions and fundamental groups, J. Pure Appl. Algebra 132 (1998), no. 2, 207-220. |MR 99i:55017

31. - A version of Zabrodsky's lemma, Proc. AMS 126 (1998), no. 5, 1573-1578. MR 98j:55011

32. K. Varadarajan, Groups for which Moore spaces $M(\pi, 1)$ exist, Annals Math. 84 (1966), 368-371. MR 34:2017

33. George W. Whitehead, Elements of Homotopy Theory, Graduate T. Math., no. 61, SpringerVerlag, Berlin New York, 1978. MR 80b:55001

Departement Mathematik, ETH, 8092 Zürich, Switzerland

E-mail address: mislin@math.ethz.ch

Department of Mathematical Sciences, University of Alberta, Edmonton, Canada T6G 2G1

E-mail address: George.Peschke@UAlberta.Ca 